\title{
Brain Tumor Segmentation using hybrid of both Netrosopic Modified Nonlocal Fuzzy C-mean and Modified Level Sets
}

\author{
Shaima Elnazer ${ }^{1}$, Mohamed Morsy ${ }^{2}$, Mohy Eldin A.Abo-Elsoud ${ }^{3}$ \\ ${ }^{1}$ Lecture Assistant, Communication Department, Nile Academy, Mans.univ, Mansoura, Egypt \\ ${ }^{2}$ Doctor, Communication Department, Mans.univ, Mansoura, Egypt \\ ${ }^{3}$ Professor, Communication Department, Mans.univ, Mansoura, Egypt
}

\begin{abstract}
Neutrosophic sets (NS) and Modified Non local Fuzzy c-mean clustering (NLFCM) is proposed. The brain tumor MRI image is transformed into NS domain, which is described using three subsets namely; the percentage of truth in a subset $T \%$, the percentage of indeterminacy in a subset $I \%$, and the percentage of falsity in a subset $F \%$. The entropy in NS is defined and employed to evaluate the indeterminacy. NS image is adapted also using Modified Non local Fuzzy Cmean algorithm(MNLFCM). Finally, MRI brain tumor image is segmented and tumor is selected using Modified Level Sets (MLS). The proposed approach denoted as NS- MNLFCM-MLS and compared with another paper using Jaccard Index and Dice Coefficient. The experimental results demonstrate that the proposed approach is less sensitive to noise and performs better on MRI brain image.
\end{abstract}

Keywords: Magnetic resonance imaging, Netrosophic, Nonlocal fuzzy c mean, Directional $\alpha$-mean operation ,modified level sets

\section{Introduction}

The Brain tumor segmentation methods can become classified into three classes in line with the level of required human being interaction as described simply by Foo et al. [1], Olabarriga et al. [2], and Yao [3]: manual segmentation, semiautomatic segmentation, and totally programmed segmentation. Brain tumors are hard to segment since they have a variety of presence and effect on adjoining structures. Following are several of the typical characteristics of brain tumors: (A) change greatly in dimensions and placement, (B) vary greatly in the way they be present in MRI, (C) might have overlapping intensities with normal tissue, (D) can be space occupying (new tissues that moves normal structure) or infiltrating (changing real estate of existing tissue), (E) may enhance fully, somewhat, or not at every, with contrast agent.

Clustering is known as a generally used mathematical procedure which in turn performs segmentation process to distinguish the structures and maternity shapes present in a great input image or dataset [4] It could be categorized into two groups: hierarchical and dividing [5].The output of hierarchical clustering are like tree and they don't need to specify the number of the amount of the clusters and independent of the first condition. However, it might fail to separate overlapping clusters due to not enough information regarding the size of clusters or its global condition. Dividing clustering algorithm on fix the disadvantage of hierarchical clustering algorithm partitions the data set in to specific number of groupings. Fuzzy based clustering allows partition an input photo into several homogenous classes or clusters, by which in turn identical conditions are grouped found in a same class and non-identical items belong to different classes.

The majority of the algorithms found in image processing are susceptible to several uncertainties, pertaining to instance, grayness ambiguity (uncertainty inside the input details itself). The key aim of this work should be to lessen uncertainty while clustering .Generally there are two main tactics in clustering technique particularly crisp and fuzzy clustering technique. Due to different situations, for images, problems like small scale of spatial resolution, poor light, occurrence of noise, power imbrications leads crisp segmentation a hard task.Between numerous clustering techniques, fuzzy c-means (FCM) [6] algorithm is usually more significant as a result of the robustness.

Although it is definitely robust it works just on the images with no noise. Many researchers experience analyzed brain MRI segmentation using FSs, see Zhao [7] Agrawal [8]. Yet these algorithms still include problems due to different situations, for instance, taking brain images under low of the illumination make it unclear. In general, FCM criteria have greater data managing capacity and have better operability after diversified info range. Due to the application of pixel neighborhood information, RFCM algorithm has nice overall performance of noise restraining, and gets good segmentation benefits comparing with standard FCM. Yet, we found that with all the increase of normal deviation of noise, the ability of noise reductions of FCM algorithm might become weaken. V. P. Ananthi [9] use Segmentation of brain tumor based on interval- respected intunistic fuzzy sets features Dice coefficient equal zero. 967. M. Zarinbal [10] work in astrocytomas extraction only using type 2 fuzzy and have got accuracy of $89 \%$.

In respect to this situation, all of us put forward clustering algorithm based in non-local information NON-LOCAL unclear C-means clustering, (NL FCM), a greater weighted neighborhood details can be used, so it is definitely better to suppress sounds than that of RFCM algorithm. Non-local 
regularization was first formerly used for picture denoising, called non-local indicate denoising [11]. The algorithm essentially uses the redundant information of normal structure, frankly neighborhood about a pixel and as well other neighbor pixels found in the same scene may match with each various other. The NLFCM algorithm identifies a weighted graph of all pixels in the image, in which the weight value is received by similarity calculation of two neighborhood pixels

The convergence rate gets affected if the number of clusters and iterations are subsequently increased. Diminishing the number of iterations and clusters to obtain faster convergence rate has an adverse effect upon the segmentation accuracy . To overcome these kinds of hindrances, a novel segmentation algorithm which combines Neutrosophy studies the foundation, characteristics, scope of neutralities, and their interactions with distinct ideational spectra. It is usually a new philosophy that extends fuzzy logic and is the basis of neutrosophic logic, neutrosophic likelihood, neutrosophic set theory, and neutrosophic statistics. Because the world is filled with indeterminacy, the imperfection of understanding that a human receives/observes from the external universe also causes imprecision. Neutrosophy introduces a new principle $<$ Neut-A $>$, which is definitely the representation of indeterminacy. However, this theory can be mostly discussed in physiology and mathematics. Thus, applications to prove this theory can resolve real challenges are needed. In this kind of dissertation, I apply neutrosophy to brain tumor segmentation. In brain tumor segmentation, neutrosophy helps reduce noises. In MRI brain tumour segmentation, eutrosophy integrates two controversial opinions about noise.

\section{Proposed Algorithm}

An superior segmentation approach based upon Neutrosophic sets (NS) and Non local Fuzzy c-mean clustering (NLFCM) is recommended. The brain tumor MRI image is become NS domain, which is defined using three subsets particularly. The entropy in NS is defined and employed to gauge the indeterminacy. NS image is modified using Moving Mean Operation (MMO) and also using Modified Not local Fuzzy C-mean algorithm (MNLFCM). Finally, MRI brain growth image is segmented and tumor is selected applying Modified Level Sets (MLS).The proposed approach denoted as NSMNLFCM-MLS and using Maxima Morphological Transform (MMT) to find accurate tumor boundary. We compared with another newspaper using sensitivity , Specificity and Dice Coefficient. The trial and error results demonstrate the fact that offered approach is less hypersensitive to noise and functions better on MRI brain image. Figure 1show the flowchart of the proposed approach.

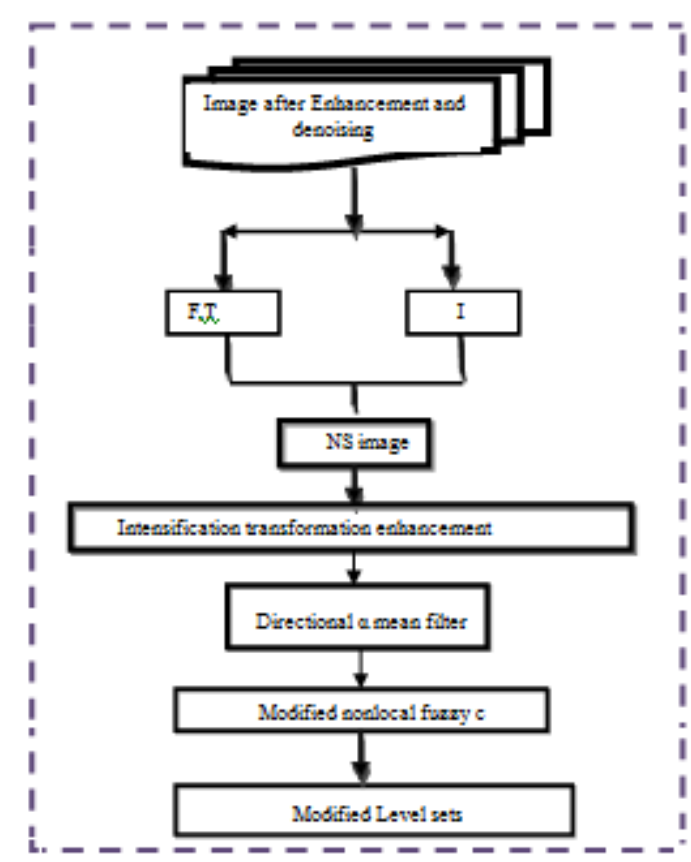

Figure 1: Step of Proposed Algorithm

\subsection{Neutrosophic MRI brain tumor Image}

Let $\mathrm{U}$ get an universe of talk, and $\mathrm{W}$ be a set included in Circumstance, which is composed simply by bright pixels. A neutrosophic image $\mathrm{P}$ is indicated by three subset To, I and F. A pixel $P$ in the image is identified as $\mathrm{P}(\mathrm{T}, \mathrm{My}$ spouse and $\mathrm{i}, \mathrm{F}$ ) and belongs to $\mathrm{W}$ in the next way: it is $\mathrm{t} \%$ true in the dazzling pixel set, i\% indeterminate, and $\mathrm{f} \%$ bogus, where $t$ varies in $T$ varies $I$, and $f$ varies in $N$. The pixel $P(i, j)$ in the image website is transformed into neutrosophic domain PNS $(i, j)=T(i, j), I(i, j), F(i, j)$. Where $T(i, j), I ~(i, j)$ and $F(i, j)$ will be the probabilities belong to bright white set, indeterminate set and non-white set, respectively [12], which are described as: equation below:

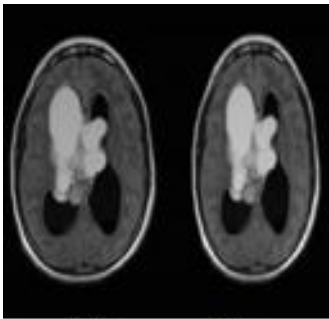

(a) (b)

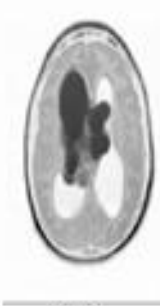

(c)

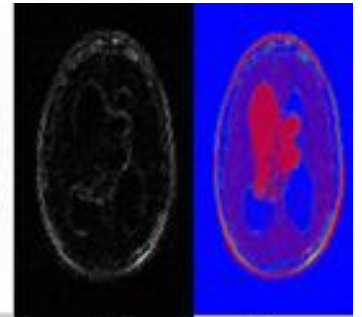

(d)

(e)
Figure 2: a) Input MRI image, b)True part. c)False part. d) Indetermance part, e) Netrosopic MRIimage

The pixel $P(i, j)$ in the image domain is transformed into Neutrosophic domain PN

$$
S(i, j)=\{T(i, j), I(i, j), F(i, j)\} \text {. }
$$

Where $\mathrm{T}(\mathrm{i}, \mathrm{j}), \mathrm{I}(\mathrm{i}, \mathrm{j})$ and $\mathrm{F}(\mathrm{i}, \mathrm{j})$ are the probabilities belong to white set, indeterminate set and non-white set, respectively, which are defined in the following equations.

$$
P_{N S}(i, j)=\{T(i, j), I(i, j), F(i, j)\}
$$

Ho $(i, j)=\operatorname{abs}(g(i, j)-g(i, j))$

$F(i, j)=1-T(i, j)$ 


\section{International Journal of Science and Research (IJSR) \\ ISSN (Online): 2319-7064}

Index Copernicus Value (2013): 6.14 | Impact Factor (2014): 5.611

$$
\begin{gathered}
\overline{g(i, j)}=\frac{1}{w \times w} \sum_{m=1-w / 2}^{1+w / 2} \sum_{n=j-w / 2}^{j+w / 2} g(m, n) \\
T(i, j)=\frac{\overline{g(i, j)}-\bar{g}_{\min }}{\bar{g}_{\max }-\bar{g}_{\min }} g(m, n) \\
I(i, j)=1-\frac{H o(i, j)-H o_{\min }}{\bar{H} o_{\max }-\bar{H} o_{\min }}
\end{gathered}
$$

Where $\overline{g(i, j)}$ is the local mean value of the image, $\mathrm{H} \mathrm{o}(i, j)$ is the homogeneity value of $\mathrm{T}$ at $(\mathrm{i}, \mathrm{j})$ which is described by the absolute value of difference between intensity g(i, j) and its local mean value $g(i, j)$. Figure show input image and if of the enhanced MRI image then NS Image are shown in fig (D)

\subsection{Enhancement of NS MRI brain tumor Image}

The MRI brain tumor picture in NS domain might increased using intensification transformation enhancement technique (ITET) to increase the top quality and emphasizes certain features of a picture to makes segmentation easier and more effective [13].Figure 3 show result of using (ITET)

$$
\begin{array}{ll}
\mathrm{E}(\mathrm{T}(\mathrm{x}, \mathrm{y}))=2 \mathrm{~T}^{2}(\mathrm{x}, \mathrm{y}) & 0 \leq \mathrm{T}(\mathrm{x}, \mathrm{y}) \leq 0.5 \\
\mathrm{E}(\mathrm{T}(\mathrm{x}, \mathrm{y}))=1-2(1-\mathrm{T}(\mathrm{x}, \mathrm{y}))^{2} & 0.5<T(\mathrm{x}, \mathrm{y}) \leq 1
\end{array}
$$

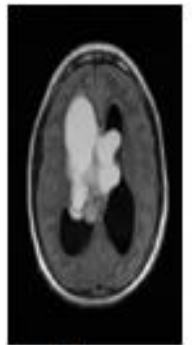

(a)

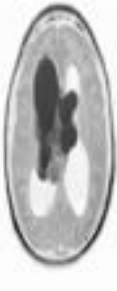

(b)

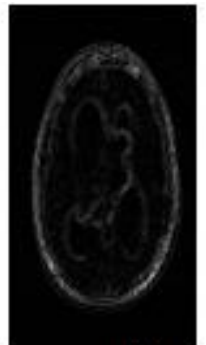

(c)

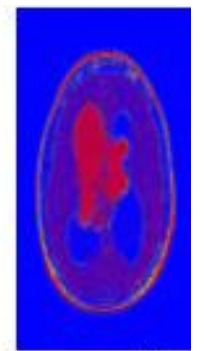

(d)
Figure 3: A) Enhanced True, b) Enhanced False. C)Enhanced Indetremnance .d) Enhanced Netrosopic Image.

\subsection{Entropy of NS brain tumor MRI Image}

Entropy is utilized to assess the distribution of diverse gray level in mind tumor MRI images. In the event that the entropy is optimum, the several intensities have even probability and the features distribute uniformly. If the entropy is small, the intensities have different possibilities and their distributions are actually non-uniform.

Definition 1 (Neutrosophic image entropy) NS picture entropy is defined while the summation of the entropies of three subsection, subdivision, subgroup, subcategory, subclass F, T and I, which is employed to evaluate the distribution in the components in NS site [14]:

$$
\begin{aligned}
\text { EnT } & =-\mathrm{PT}(\mathrm{i}) \ln \mathrm{PT}(\mathrm{i}) \\
\text { EnF } & =-\mathrm{PF}(\mathrm{i}) \ln \mathrm{PF}(\mathrm{i}) \\
\text { EnI } & =-\mathrm{PI}(\mathrm{i}) \ln \mathrm{PI}(\mathrm{i})
\end{aligned}
$$

EnNS=EnT+EnI+EnF (12) Where EnI , EnT and EnF are the entropy of subsets $\mathrm{T}$, I and $\mathrm{F}$, respectively as shown in fig 4 .

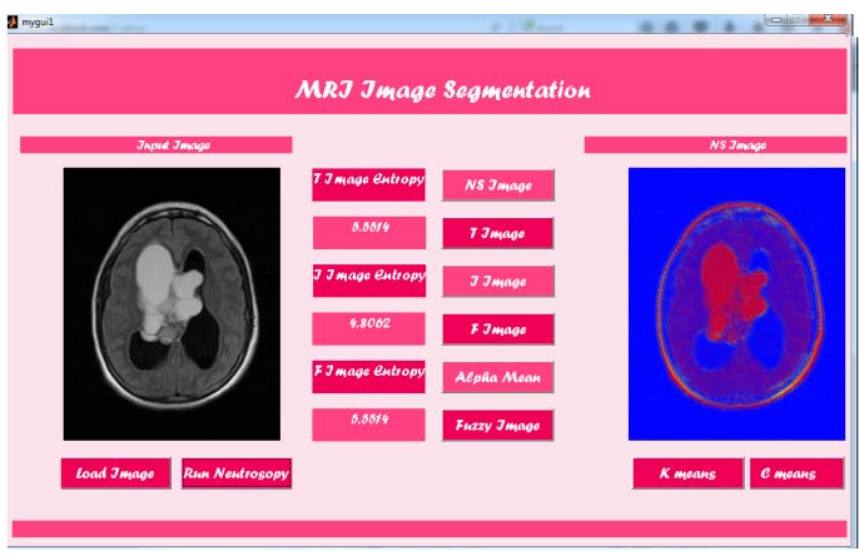

Figure 4: GUI Result of entropy calculation

\subsection{Directional $\alpha$-mean operation}

In [14], an $\alpha$-mean functioning was defined on a neutrosophic image, and it taken off noise efficiently However, it could blurring the image and lessen the contrast, which can reduce the performance of the segmentation to overcome this kind of drawback, directional $\alpha$-mean operation denoted as DAM is usually newly proposed to remove the noise effect and conserve the edges at the same time. The function of the directional mean filter DAM is identified as [16]:

$$
\operatorname{DMF}(i, j)= \begin{cases}R_{1} & \left|G_{T_{h}}(i, j)-G_{T v}(i, j)\right| \leq \sigma \\ R_{2} & G_{T_{b}}(i, j)-G_{T_{v}}(i, j)>\sigma \\ R_{1} & G_{T_{v}}(i, j)-G_{T_{h}}(i, j)>\sigma\end{cases}
$$

Where G (i, j) Th and G(i, j) TV are the norm of the gradientat (,at the horizontal and vertical direction, respectively.

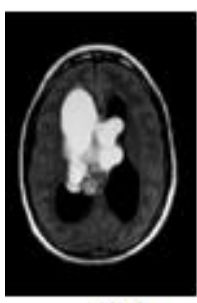

(a)

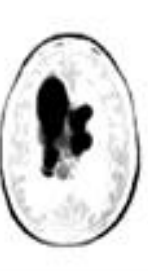

(b)

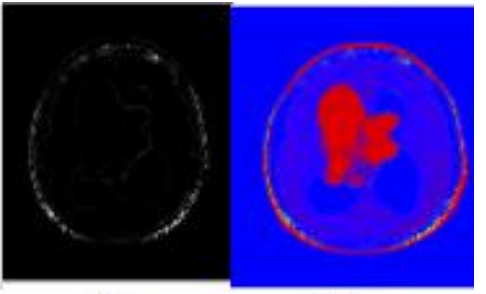

(c) (d)
Figure 5: Directional mean enhancement of :a)T,b)I.c)F.d)NS

\subsection{Modified Non Local Fuzzy C mean :}

As shown in above, NON local FCM (NLFCM) is extremely sensitive to the outliers. The membership function of the Nonlocal unclear is modified by looking at the outliers rejection; Frequency. (10) becomes: 16

$J_{R F C M}=\underset{j \in \Omega}{\infty} \sum_{k=1}^{C} u_{j k}^{q}\left\|y_{j}-v_{k}\right\|^{2}+\frac{\beta}{2} \sum_{j \in \Omega} \sum_{k=1}^{C} u_{j k}^{q} \sum_{n \in N_{j}^{k}} w_{j n} \sum_{l \in L_{k}} u_{n l}^{q}$

The part of the exponent is definitely to limit the partially distribution with the points between two neighboring clusters somewhat than to all groupings. Is defined as: 


\section{International Journal of Science and Research (IJSR) \\ ISSN (Online): 2319-7064}

Index Copernicus Value (2013): 6.14 | Impact Factor (2014): 5.611

$$
\alpha=\frac{\left(\mathrm{X}_{\max }-\mathrm{X}_{\min }\right)+1}{256}+1
$$

Exactly where $\mathrm{X}$ max and Times min are respectively, the maximum and the minimal intensity in the image is between 1 and 2 If the graphic has a big intensity selection the partial distribution of the pixels between 2 surrounding clusters is reduced. Not likely, if the image includes a small intensity range, is usually close to 1 and the partial distribution of the pixels is basically among the adjacent groupings.

The membership function of NLFCM is modified by simply replacing the original length from equation 8 in [11]

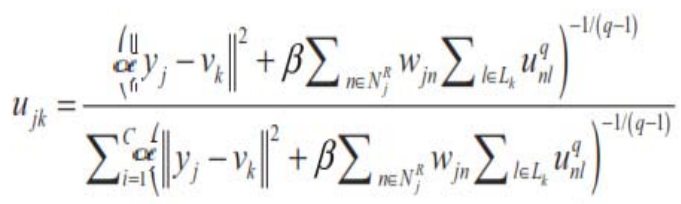

Combining NS brain tumor image with MNLFCM we get accurate place of brain tumor as shown in figure 6 .
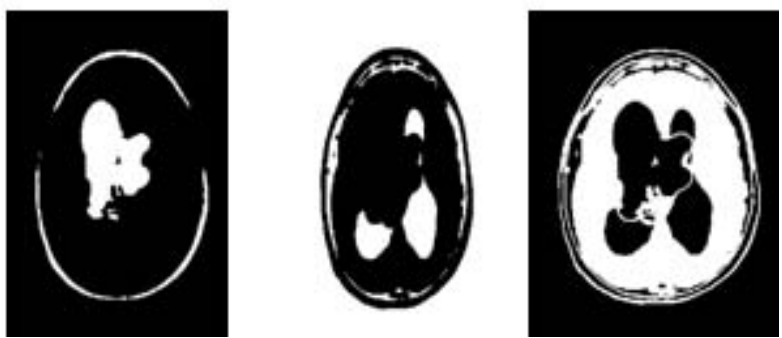

Figure 6: Clustering of Netrosophic image using MNLFCM

\subsection{Modified level sets}

After using netrosophic with Modified Nonlocal Fuzzy C Mean (MNLFCM) we have to make use of boundary method to get accurate tumor. In General, picture segmentation models using level sets method(LSM )can always be classified as edge-based types or region-based models [17][18]. The former utilizes edge details [19] although the latter employs a region descriptor to control the displacement of the active contour [20], [21]. Edge-based types are not sensitive to inhomogeneity of image features. But are sensitive to objects with poorly described tumors boundaries. In images in which the intensities change steadily in the vicinity of a poorly defined border, the border-stop function (BSF) does not stop the contour [22].

To overcome the limitation with the traditional BSFs in border based active contour types, we propose a construction to construct a group of robust BSFs that make use of probability scores rather than the predicted class brands from a classifier. Seeing that the scores fall in $[0,1]$, this kind of task is just like fuzzy segmentation. Unlike the techniques of [23], [24], which in turn rely only on category probability using Bayes' guideline, our framework is considerably more flexible since it utilizes the probability scores by any classifier. At the same time, we maintain gradient information to end contour evolution when presently there are no fuzzy principles due to distinct limitations. These ideas differentiate the work from [25], which relies on energy and is also considered a region-based level set technique. The traditional BSF needs reinititialization to avoid irregularities during its evolution [20], [21]. As reinitialization often brings about problems, Li et al. [22] proposed the length regularized level set development (DRLSE) which removes the advantages of reinitialization. That they applied the DRLSE to an edge-based active curve model by introducing the gradient flow as equation(19)

$$
\begin{aligned}
\frac{\partial \phi}{\partial t}= & \mu \operatorname{div}\left(d_{p}(|\nabla \phi|) \nabla \phi\right) \\
& +\lambda \delta_{\epsilon}(\phi) \operatorname{div}\left(g \frac{\nabla \phi}{|\nabla \phi|}\right)+\beta g \delta_{\epsilon}(\phi)
\end{aligned}
$$

We use the fuzzy ESF

$$
\beta=(2(P-0.5))^{2}
$$

Where $\mathrm{P}$ is the probability score for the foreground Subsequently, the fuzzy ESF is used to regularize function in (4) to obtain which can be simply expressed by

$$
\mathrm{g}_{\text {new }}=g \beta
$$

The fuzzy ESF, will compel to be near 0 when is near zero despite the fact that is much higher than 0, i.e., when the picture force drops bit by bit .Consequently, will be near 0 which will stop a shape at the fancied limit. It is clear that capacity assumes an imperative part when an inadequately characterized limit is available. It produces a base worth when the scores are at the choice limit. Besides, holding the inclination data is helpful at clear limits subsequent to there are no fluffy qualities. Capacity fuses both of these favorable circumstances to give exact division results. What's more, the proposed structure is adaptable

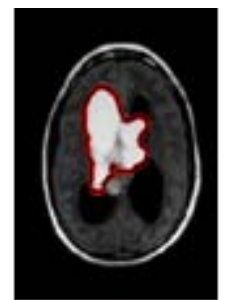

(a)

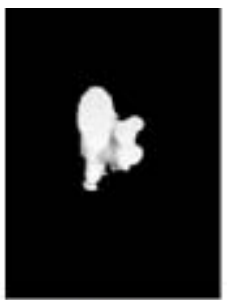

(b)
Figure 7: a) Modified level sets b) Segmented tumor part

\subsection{Maxima transform Enhancement}

For an graphic is definitely made using morphological operations such as iterated dilations of the image and later it is camouflaged. Koh et al [29] have defined $\mathrm{H}$ transform as where RI (I - h) is the retraced image by dilating I with respect to $\mathrm{I}$ - h. This sort of restrains all pixels in whose power rate is littler than the limit if maybe the worth is rather than their neighbors. Well then locate all territorial ideal of picture and individual the pixels of successive force. Maxima strategy is taken out utilizing the equation Regional maxima of H-maxima changed group picture having tumor can be utilized to uproot neighborhood pixels of power underneath $h$ from the capabilities. Utilizing Eq. (9), last particular tumor locale is unquestionably separated without edema and other non-tumor area. H-maxima change happen to be shown in Fig.8.

$$
\begin{aligned}
& H_{\text {max }_{h}}(I)=R_{I}(I-h) \text {, } \\
& T_{\max h_{h}}(I)=R_{\max }\left(H_{\max _{h}}(I)\right)
\end{aligned}
$$




\section{International Journal of Science and Research (IJSR)}

ISSN (Online): 2319-7064

Index Copernicus Value (2013): 6.14 | Impact Factor (2014): 5.611

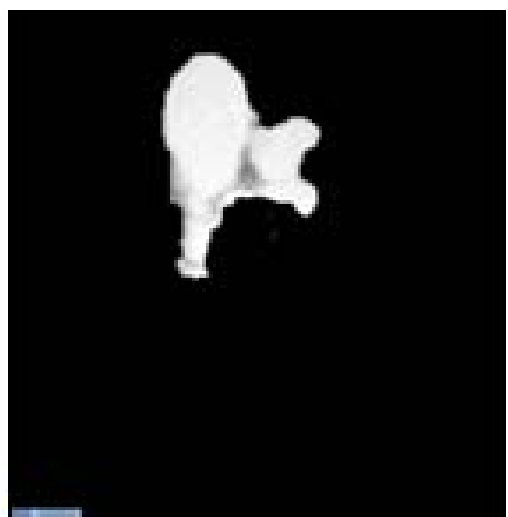

Figure 8: Segmented tumor using $\mathrm{H}$ transform

\subsection{Performance Evaluation Metric:}

The datasets for experimental analysis were obtained from Mansoura Hospital University MRI Scan. Tests are executed on numerous brain MR image datasets having tumor. To evaluate the segmentation accuracy, we used three metrics, namely, (i) the Dice Similarity Coefficient (DSC), (ii) the 98percentile modified Hausdorff distance (H98)[30]. The following subsections will describe each used metric in more detail.

A) Dice Similarity Coefficient The Dice similarity coefficient (DSC) measures set agreement between two sets $(S, G)$, and is defined as the union size of the two sets divided by the average size of the two sets

$$
\operatorname{DSC}(S, G)=(2|S \cap G| /(S \cap G+S \cup G)) \times 100
$$

In segmentation validation, the DSC is usually expressed in terms of false positive (FP), false negative (FN), true negative (TN), and true positive (TP) counts, which were obtained by comparing the segmentation results to the ground truth (gold standard) (see Figure 31). These values can be used to calculate the DSC as shown by [30]:

$$
\mathrm{DSC}=2 \mathrm{~T} \mathrm{P} /(2 \mathrm{~T} \mathrm{P}+\mathrm{F} \mathrm{P}+\mathrm{F} \mathrm{N}) \times 100
$$

A DSC estimation of zero shows no cover; an estimation of one demonstrates perfect division. Higher numbers show better division, which implies that the division results coordinate the ground truth superior to anything results with lower qualities DSC values.

Table 1: Accuracy of the proposed segmentation approach for each subject using dice similarity coefficient (DSC(\%)). \&Accuracy of the proposed segmentation approach for each subject using the 98 percentile modified Hausdorff distance

\begin{tabular}{|c|c|c|}
\hline Patient number & DSC & Hausdroff \\
\hline p.1 & 98.403 & 2.05 \\
\hline P.2 & 96.56 & 5.13 \\
\hline p.3 & 97.34 & 1.78 \\
\hline p.4 & 96.76 & 12.43 \\
\hline
\end{tabular}

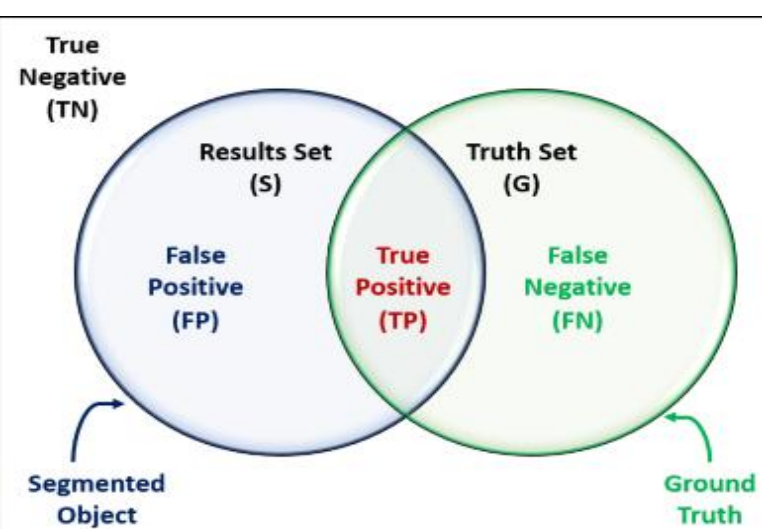

Figure 9: Diagram illustrating the meaning of segmentation errors, namely, true positive (TP), false positive (FP), true negative (TN), and false positive (FP). These segmentation errors, obtained by comparing the segmented and the ground

truth objects, are used to calculate the dice similarity coefficient (DSC).

\section{B) Modified Hausdorff Distance}

Distance measures are another type of performance metric used for evaluating segmentation methods. The Euclidean distance is often utilized, but another common measure is the Hausdorff distance $(H)$. The $H$ value from a set $S$ to a set $G$ is defined as the maximum distance of the set $S$ to the nearest point in the set $\mathrm{G}$ (see Figure 6 [30]):

$$
\mathrm{H}(\mathrm{S}, \mathrm{G})=\operatorname{maxs} \in \mathrm{S}\{\operatorname{ming} \in \mathrm{G}\{\mathrm{d}(\mathrm{s}, \mathrm{g})\}\} \text {, }
$$

where s and g are points of sets S and G, respectively, and $\mathrm{d}(\mathrm{s}, \mathrm{g})$ is Euclidean distance between these points. The bidirectional Hausdorff distance, denoted by $\mathrm{H}$ (S, G), between the segmented region (S) and its ground truth $(G)$ is defined as:

$$
\operatorname{HBi}(S, G)=\max \{H(S, G), H(G, S)\} .
$$

In this paper, to eliminate the effect of segmentation outliers, the 98-percentile modified Hausdorff distance ( $\mathrm{MH}$ ) was used to assess the proposed segmentation framework accuracy. Metrics were computed by comparing a ground truth segmentation to results from the proposed segmentation technique. The detailed segmentation results for each subject are given in Tables 1

As demonstrated in Table 1, the DSC for segmentation of the brain tumor is gives good result using our proposed algorithm .Performance shown in fig (9) and in table2.

\section{Conclusion}

In this paper, the proposed full automatic brain tumor segmentation technique has been denoted as NS- MNLFCMMLS and using Maxima Morphological Transform (MMT) to find accurate tumor boundary. It is based on neutrosophic preprocessing method and Modified non local fuzzy c mea Clustering method Compared with another new paper using Jaccard Index and Dice Coefficient. Results demonstrate the fact that offered approach is less hypersensitive to noise and functions better on MRI brain image .Proposed algorithm give high accuracy result compared with another methods. The results of the proposed method show that the $100 \%$ detection rate in all 34 cases with average of high dice

\section{Volume 5 Issue 3, March 2016}




\section{International Journal of Science and Research (IJSR) \\ ISSN (Online): 2319-7064}

Index Copernicus Value (2013): 6.14 | Impact Factor (2014): 5.611

99.37\%, high specificity 99. 26\% and lower missing rate 0.52, and modified Hausdroff distance 1.302.

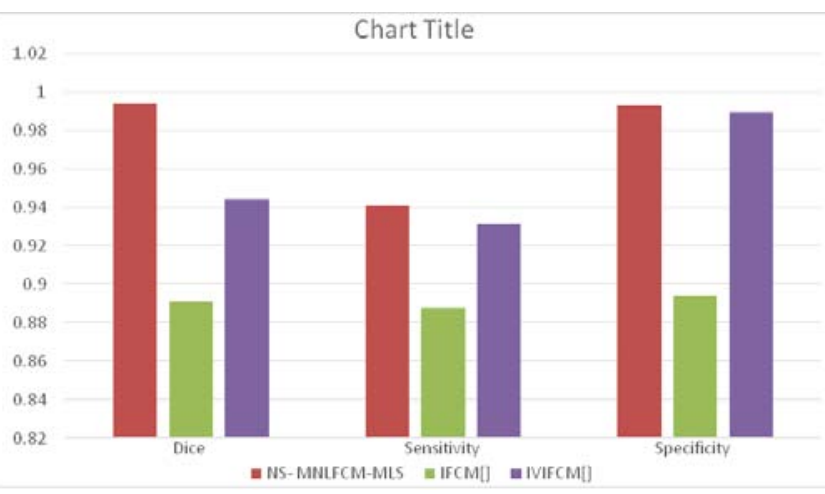

Figure 9: Comparison of proposed algorithm and paper[9],[10]

Table 2: Accuracy of the proposed segmentation approach for each MRI brain tumor for 34 patient using dice, sensitivity and specitivity \&Accuracy of the proposed segmentation approach for each subject using the 98 percentile modified Hausdorff distance

\begin{tabular}{|c|c|c|c|}
\cline { 2 - 4 } \multicolumn{1}{c|}{} & Dice & Sensitivity & Specificity \\
\hline NS- MNLFCM-MLS & 0.9937 & 0.9412 & 0.9926 \\
\hline IFCM[9] & 0.8908 & 0.8876 & 0.894 \\
\hline IVIFCM[10] & 0.944 & 0.9314 & 0.9892 \\
\hline
\end{tabular}

\section{Reference}

[1] Foo JL. A survey of user interaction and automation in medical image segmentation methods. Tech rep ISUHCI20062, Human Computer Interaction Department, Iowa State Univ; 2006.

[2] Olabarriaga S, Smeulders A. Interaction in the segmentation of medical images: a survey. Med Image Anal 2001;5:127-42.

[3] Yao J. Image processing in tumor imaging. New techniques in oncologic imaging; 2006. p. 79-102.

[4] Rafael C. Gonzalez and Richard E. Woods, Digital Image Processing, Second Ed.., Pearson Education publication, ISBN 81 - 7808 - 629 - 8, pp.68 - 70 .

[5] G.R. Sinha and Bhagawati Charan Patel, Medical Image Processing: Concepts and Applications, PHI publications, ISBN-978-81-203-4902-5, 2014.

[6] Zhou D, Zhou H ,A modified strategy of fuzzy clustering algorithm for image segmentation. 2014.Soft Comput, pp 1-12.

[7] Zhao F, Jiao L, Liu H (2013) Kernel generalized fuzzy c-means cluster-ing with spatial information for image segmentation. Digit Signal Process. 23:184-199

[8] Agrawal S, Panda R, Dora L (2014) A study on fuzzy clustering for magnetic resonance brain image segmentation using soft computing approaches. Appl Soft Comput 24:522-533

[9] V. P. Ananthi,A new fuzzy clustering algorithm for the segmentation of brain tumor Springer-Verlag Berlin Heidelberg 2015

[10] M. Zarinbal,Interval type-2 fuzzy image processing expert system for diagnosing brain tumors. M. Zarinbal ; Dept. of Ind. Eng., Amirkabir Univ. of Technol., Tehran,
Iran Norbert Wiener in the 21st Century (21CW), 2014 IEEE Conference

[11] A Fuzzy c-Means Clustering Scheme Incorporating NonLocal Spatial Constraint for Brain Magnetic Resonance Image Segmentation Cong, Wang; Song, Jianhua; Wang, Lei; Liang, Hong; Li, Jin .Journal of Medical Imaging and Health Informatics, Volume 5, Number 8, December 2015, pp. 1821-1825(5)

[12] Mohan, J., Krishnaveni, V., Guo, Y., Kanchana, J.: MRI denoising based on neutrosophic wiener filtering. In: IEEE International Conference on Imaging Systems and Techniques (IST), pp. 327-331 (2012)

[13]Zhang, M., Zhang, L., Cheng, H.D.: A neutrosophic approach to image segmentation based on watershed method. Signal Processing 90, 1510-1517 (2010)

[14]Guo, Y., Cheng, H.D., Zhang, Y., Zhao, W.: A new neutrosophic appraoch to image thresholding. In: Proceedings of the 11th Joint Conference on Information Sciences, pp. 1-6. Atlantis Press (2008)

[15] Y. Guo and H. D. Cheng, "New neutrosophic approach to image segmentation," Pattern Recognition, vol.42, pp. 587595 ,

[16] Y. Guo, H. D. Cheng, J. Tian and Y. Zhang. A novel approach to speckle reduction in ultrasound imaging. Ultrasound in Medicine \& Biology, vol.35, 2009.

[17] C.Li,R.Huang,Z.Ding,J.Gatenby,D.N.Metaxas,andJ.C.G ore, "A level set method for image segmentation in the presence of intensity inhomogeneities with application to mri," IEEE Trans. Image Process.,vol. 20, no. 7, pp. 2007-2016, 2011.

[18] S. Mukherjee and S. Acton, "Region based segmentation in presence of intensity inhomogeneity using legendre polynomials," IEEE Signal Process. Lett., vol. 22, no. 3, pp. 298-302, Mar. 2015.

[19] V. Caselles, R. Kimmel, and G. Sapiro, "Geodesic active contours,” Int. J. Comput. Vis., vol. 22, no. 1, pp. 61-79, 1997.

[20]D. Mumford and J. Shah, "Optimal approximations by piecewise smooth functions and associated variational problems," Commun. Pure Appl. Math., vol. 42, no. 5, pp. 577-685, 1989.

[21]T. F. Chan and L. Vese, "Active contours without edges," IEEE Trans. Image Process., vol. 10, no. 2, pp. 266-277, 2001.

[22] A. Pratondo, B. P. Nguyen, C.-K. Chui, and S.-H. Ong, "Vocal cord segmentation from CT images using machine learning," in Proc. $10^{\text {th }}$ Asian Conf. Computer Aided Surgery (ACCAS 2014), 2014, pp. 40-41, The Japan Society of Computer Aided Surgery.

[23] J. Wu, Z. Yin, and Y. Xiong, "The fast multilevel fuzzy edge detection of blurry images," IEEE Signal Process. Lett., vol. 14, no. 5, pp. 344-347, 2007.

[24] D. Smeets, D. Loeckx, B. Stijnen, B. De Dobbelaer, D. Vandermeulen, and P. Suetens, "Semi-automatic level set segmentation of liver tumors combining a spiralscanning technique with supervised fuzzy pixel classification," Med. Image Anal., vol. 14, no. 1, pp. 1320, 2010.,

[25] S. Krinidis and V. Chatzis, "Fuzzy energy-based active contours," IEEE Trans. Image Process., vol. 18, no. 12, pp. 2747-2755, 2009., 
[26]S. Osher and R. Fedkiw, Level Set methods and Dynamic Implicit Surfaces,S.S.Antman,J.E.Marsden,andL.Sirovich,Eds. NewYork, NY, USA: Springer-Verlag, 2003.

[27] J. A. Sethian, Level Set Methods and Fast Marching Methods: Evolving Interfaces in Computational Geometry, Science,P.G.Ciarlet,A.Iserles,R.V.Kohn,and M. H. Wricht, Eds. New York, NY, USA: Cambridge Univ. Press, 1999, vol. 3.

[28] C.Li,C.Xu,C.Gui,andM.D.Fox, "Distanceregularizedlevel setevolution and its application to image segmentation," IEEE Trans. Image Process., vol. 19, no. 12, pp. 32433254, Dec. 2010.

[29] Koh KH, Shen WA, Shuter B, Kassim AA (2009) Segmentation of kidney cortex in MRI studies: a constrained morphological 3D h-maxima transform approach. Intern J Med Eng Inf 1:330-341

[30] K. O. Babalola, B. Patenaude, P. Aljabar, J. Schnabel, D. Kennedy, W. Crum, S. Smith, T. Cootes, M. Jenkinson, and D. Rueckert. An evaluation of four automatic methods of segmenting the subcortical structures in the brain. Neuroimage, 47(4):1435-1447, 2009. 